\title{
Communication
}

\section{Drawing Electrochemical Sensors Using a 3D Printing Pen}

\author{
Rafael M. Cardoso, ${ }^{*, a}$ Sílvia V. F. Castro, ${ }^{\circledR a}$ Jéssica S. Stefano ${ }^{\circledR a}$ and \\ Rodrigo A. A. Muñoz ${ }^{\circledR * a}$
${ }^{a}$ Núcleo de Pesquisa em Eletroanalítica, Instituto de Química, Universidade Federal de Uberlândia, 38408-902 Uberlândia-MG, Brazil

\begin{abstract}
This communication demonstrates that a 3D printing pen, commercially-available as a toy for kids, can be used to draw thermoplastic electrochemical sensors using conductive filaments over any desirable format and substrate. A polylactic acid filament containing graphene or carbon black was passed through the 3D pen that works as a handheld 3D printer (fused deposition modeling type). As proof-of-concepts, we select three designs, the first one analogous to a conventional carbon-paste electrode, the second one containing the three electrodes in a cylindrical tube and the third one similar to commercial screen-printed electrodes. The 3D pen was used to draw the thermoplastic electrodes inside the pockets of all devices until reaching the copper wires to establish electric contact. Customized 3D printed substrates were used to guide the application of the 3D pen. The fabricated electrodes were applied for the detection of dopamine and trace metals as well as to generate electrochemically a Prussian Blue modified sensor. Many other designs, conductive filaments and substrates can be combined with a 3D pen to draw electrochemical sensors as well as other electrochemical devices, with applications ranging from medical point-of-care platforms, energy-storage devices as well as for teaching purpose.
\end{abstract}

Keywords: 3D printing, additive manufacture, disposable electrodes, portable devices, fused deposition modeling, graphene

\section{Introduction}

Additive manufacturing or three-dimensional (3D) printing has created a revolution in several areas, including medical, electronics, mechanical engineering, civil engineering, food, space exploration, and chemical industry. ${ }^{1}$ In the chemistry area, the 3D-printing technology has been widely applied to the fast prototype of reactionware, ${ }^{2}$ microfluidic devices, ${ }^{3}$ supercapacitors, ${ }^{4,5}$ batteries, ${ }^{6,7}$ electrochemical sensors, ${ }^{8-14}$ smartphone-based analytical devices, ${ }^{15,16}$ and water splitting processes. ${ }^{7,17,18}$ Different 3D-printing techniques are available and probably the most popular is the fused deposition modeling (FDM). Its popularity is due to open-source code philosophy, where by following tutorials available for free and low-cost accessible parts, the budget for the construction of such machine can drop to values of less than U\$100 (R\$550). Thus, FDM 3D-printers can be considered a personal equipment to prototype any object anywhere. Recently, FDM 3D-printers have become a very important tool for the fast fabrication

*e-mail: rcardosoq@gmail.com;munoz@ufu.br of face shield masks for medical staff working against COVID-19 (coronavirus disease 2019) as an on-demand strategy of emergency tool for plastic manufactured parts. ${ }^{19}$

Another simpler and non-automated handheld version of an FDM 3D-printer is a 3D pen, commonly used as a toy for children. The 3D pen extrudes a thermoplastic filament under controlled speed and temperature started by the user by pressing a button at the pen. The difference of a 3D pen to an FDM 3D-printer is that the application of the molten thermoplastic filament is made by the user's hand while the 3D printer works in a mechanized way. Although an FDM 3D-printer is a low-cost instrument with an average of cost of U\$250, a 3D pen has a 10-fold lower price (around U\$25).

When applied to prototype electrochemical devices such as batteries, supercapacitors and electrochemical sensors, conductive thermoplastic filaments are imperative. ${ }^{4,5}$ Commercially-available sources based on polylactic acid (PLA) can be found. The most common conducting agents in these composites are graphene or carbon black. ${ }^{20,21}$ Lab-made conductive filaments have also been proposed for 3D-printing for several applications, including 
electrochemical sensors, supercapacitor and batteries in which the conductivity of the filaments for 3D printing was dramatically improved by using a high carbon content (graphite or carbon black) or carbon nanotubes. ${ }^{22-26}$

Recently, a 3D printing pen was proposed to fabricate bioactive materials for medical application ${ }^{27}$ and to fabricate paper-based analytical devices (create polymeric barriers on paper devices for colorimetric detection). ${ }^{28}$ This device can be considered as a handheld FDM 3D-printer that can extrude thermoplastic filaments. In this context, this communication demonstrates that a similar 3D printing pen can be used to manufacture electrochemical devices by introducing conductive filaments through the pen. Customized 3D printed substrates were developed to guide the application of the molten filament using the 3D pen. We selected three different designs to fabricate electrochemical sensors in which the conductive part of the electrode is the thermoplastic carbon-based material drawn by the 3D pen.

\section{Experimental}

Deionized water (resistivity not lower than $18.2 \mathrm{M} \Omega \mathrm{cm}$ ) obtained from a Milli-Q water purification system (Millipore, Bedford, MA, USA) was used to prepare all solutions. All reagents were analytical grade and used with no further purification: dopamine (>99\%) from Acros (New Jersey, USA), perchloric acid (70\%) from Reagan (Rio de Janeiro, Brazil), potassium ferrocyanide from CAAL (São Paulo, Brazil), and potassium ferricyanide from Proquimios (Rio de Janeiro, Brazil). Hydrochloric acid ( $37 \% \mathrm{~m} / \mathrm{v}$ ) and potassium chloride was obtained from Synth (Diadema, Brazil). Aqueous standard solutions of antimony and lead (1000 mg L ${ }^{-1}$ ) were obtained from Quimlab (Jacareí, Brazil). Sodium hydroxide was obtained from AppliChem Panreac (Barcelona, Spain), dimethylformamide (DMF) from Merck (Rio de Janeiro, Brazil) and hydrogen peroxide (30 wt.\%) from Dinâmica (São Paulo, Brazil).

A 3D pen obtained from Sanmersen (Shenzhen, China) was used to fabricate the electrochemical sensors using conductive filaments made of polylactic acid (PLA) doped with graphene (Black Magic ${ }^{\circledR}$ purchased from BlackMagic3D, Ronkonkoma, NY, USA) or doped with carbon black (Proto-Pasta ${ }^{\circledR}$ obtained from ProtoPlant Inc., Vancouver, Canada). This device is typically used as a toy for children and can be considered a handheld FDM 3D-printer. The pen contains a geared mechanism assembled for pushing filament along a filament guide tube towards a heated nozzle. The nozzle temperature was kept at $190{ }^{\circ} \mathrm{C}$. Other 3D pen models enable controlled temperature variation. When the filament reaches the high temperature of the nozzle at the end of the pen, the filament fuses and can be manually extruded as the molten plastic rapidly solidifies at room temperature. Figure 1A shows a scheme of the 3D pen and its components. Figure 1B shows three different designs fabricated using FDM 3D printing to illustrate possible applications for electrochemical sensors. Figure $1 \mathrm{Ba}$ (in blue) shows the representation of side and top views of an electrode inside a cylindric tube similar to a conventional carbon-paste electrode. The extruded conductive filaments by the 3D pen were applied into the empty pocket covering the copper wires (black region at the image). Figure $1 \mathrm{Bb}$ (in gray) shows the representation of front view and three side views of the same device, which contains three electrodes inside a tube, all of them connected to independent copper wires. The three empty pockets in the customized 3D printed substrate are filled with the extruded conductive filaments by the 3D pen. Figure 1Bc (in light green) shows the third design (top view and two side views) similar to a planar screen-printed electrode in which three copper wires are concentrically passed through the plastic substrate to reach empty pocket filled with the conductive filaments by the $3 \mathrm{D}$ pen. The customized substrates of the three designs were fabricated using a desktop FDM 3D-printer with a non-conductive PLA filament and at the back side was introduced a copper wire to establish the external electric contact of each electrode. A video is presented in the Supplementary Information (SI) showing the step-by-step fabrication of the device shown in Figure 1Bc. After the application of the conductive filament using the $3 \mathrm{D}$ pen that solidifies in less than $5 \mathrm{~s}$, the obtained device was polished using an electric mini drill equipped with a sanding paper attached to a rubber wheel (as seen in the video) to remove the excess of conductive filament over the planar surface. Before electrochemical measurements, the device was polished on a clean polishing pad wet with deionized water. The substrates used to insulate the working electrode and guide the application of the molten conductive filaments using the 3D pen can be fabricated using other materials, such as Teflon (commonly used in commercial glassy carbon disc electrodes), acrylic, cured resins, among others.

The pseudo-reference electrode of the design presented in Figure $1 \mathrm{Bc}$ was modified with $\mathrm{Ag} / \mathrm{AgCl}$ to produce a more stable pseudo-reference electrode using a protocol described in the literature. ${ }^{29}$ Briefly, the exposed carbon black thermoplastic surface was electrodeposited with $\mathrm{Ag}$ from a silver nitrate solution commercially available as PLCSP-100 silver-plating solution (typically used for copper protection in electronic components) purchased from PLC Comércio de Placas e Componentes Eletrônicos (Sao Paulo, Brazil), by applying -0.9 V versus 


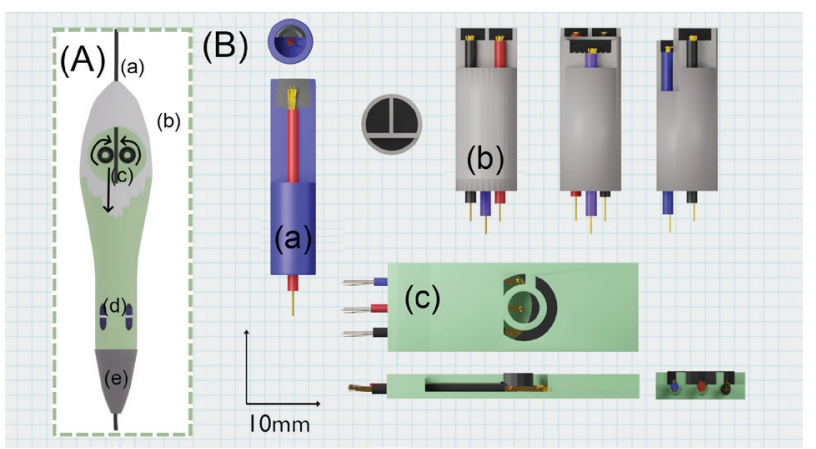

Figure 1. (A) Simplified representation of internal parts of the 3D pen: (a) conductive thermoplastic filament; (b) geared mechanism; (c) filament guide tube; (d) command buttons; (e) heated nozzle $\left(190^{\circ} \mathrm{C}\right)$; (B) scaled versions of the proposed devices: (a) single working electrode (side and top views); (b) three electrodes inside a tube (front view and three side views) with independent copper wires; (c) planar device with three electrodes (top view and two side views) with independent copper wires. Black regions correspond to the pockets (holes) filled by the molten conductive filament.

$\mathrm{Ag}|\mathrm{AgCl}| \mathrm{KCl}_{\text {sat }}$ for $800 \mathrm{~s}$ and using an external Pt wire as the counter electrode. Next, an aliquot of a commercial chlorine bleach solution (3\%, Start Química, Uberlândia, Brazil) was dropped over the Ag-plated electrode to form the pseudo-reference $\mathrm{Ag} / \mathrm{AgCl}$ electrode.

Electrochemical measurements were performed at room temperature and without oxygen removal from solutions. A micro-AUTOLAB type III potentiostat/galvanostat (Metrohm Autolab B. V., Utrecht, Netherlands) interfaced to a microcomputer and controlled by NOVA 1.12 software was used to obtain and register the measurements. A 3D-printed electrochemical cell was manufactured using ABS filament (1.75 mm) and a fused deposition modelling 3D-printer GTMAX 3D (São Paulo, Brazil) according to the literature. ${ }^{30}$ This cell was used for experiments using conventional three-electrode system (Pt wire and $\left.\mathrm{Ag}|\mathrm{Ag}| \mathrm{KCl}_{\text {sat }}\right)^{31}$ in combination with the cylindrical 3D pen printed working electrode presented in Figure 1Ba. Some experiments using the devices presented in Figures $1 \mathrm{Bb}$ and $1 \mathrm{Bc}$ were performed using this lab-made external $\mathrm{Ag}|\mathrm{AgCl}| \mathrm{KCl}_{\text {sat }}$ instead of the pseudo-reference already presented in the device for comparison purposes.

The working electrode, consisting of a thermoplastic conductive PLA (either with carbon-black or graphene as the conductive agent), requires a surface treatment to make more available the condutive sites within the polymeric matrix. Based on the literature, two different protocols were used dependending on the conductive PLA used in the 3D pen fabrication of the electrodes. Graphene/PLA electrodes were treated by mechanical polishing followed by immersion in DMF for $10 \mathrm{~min} .{ }^{32}$ Carbon black/PLA electrodes were electrochemically treated by a sequence of applied potentials $(+1.4 \mathrm{~V}$ for $200 \mathrm{~s}$ and $-1.0 \mathrm{~V}$ for $200 \mathrm{~s})$ using $\mathrm{Ag}|\mathrm{AgCl}| \mathrm{KCl}_{\text {sat }}$ as reference electrode and a platinum wire as counter electrode in a $0.5 \mathrm{~mol} \mathrm{~L}^{-1} \mathrm{NaOH}$ solution as the supporting electrolyte. ${ }^{33,34}$

\section{Results and Discussion}

The results presented herein aimed to demonstrate the feasibility of the electrochemical sensors fabricated with the aid of a 3D pen. The minimum thickness using the 3D pen is $0.69 \pm 0.01 \mathrm{~mm}(\mathrm{n}=3)$ and its use is dependent on the user hability to handle the $3 \mathrm{D}$ pen to draw the desired 3D object. For this reason, we proposed customized 3D printed substrates to guide the application of the molten conductive filament using the 3D pen and consequently to increase the final precision of the fabricated electrodes. The 3D printed substrates shown in Figure 1B were based on conventional electrochemical sensing devices.

The use of desktop 3D printers to fabricate thermoplastic electrochemical sensors has already been reported in the literature ${ }^{8-14}$ and the surface treatment of the fabricated electrodes provides dramatic improvement of their sensing properties. The excess of PLA and difficult access to the conductive sites of graphene or carbon black hinder the electrochimical activity of such electrodes. Hence, a surface treatment of the fabricated electrode is required and we selected an electrochemical procedure in alkaline medium due to easy access to $\mathrm{NaOH}$ in the case of conductive PLA filaments containing carbon black and solvent immersion of the electrodes in DMF in the case of conductive PLA filaments containing graphene. The process using $\mathrm{NaOH}$ solution for PLA degradation accelerated by the electrochemical process is advantageous due to its simplicity. ${ }^{33,34}$ Electrode immersion for $10-30 \mathrm{~min}$ in a solvent (acetone or dimethylformamide) ${ }^{12,35}$ is also a simple process. Other procedures reported in the literature such as simple mechanical polishing ${ }^{36}$ or thermal treatment ${ }^{37}$ can be evaluated for this aim.

Figure 2 shows an application of the cylindrical embedded electrode (Figure 1Ba), in which a graphene/ PLA filament was extruded by the 3D pen, for the determination of $\mathrm{Pb}^{2+}$ and $\mathrm{Sb}^{3+}$ using square-wave anodic stripping voltammetry (SWASV). This technique involved three sequential steps: (i) electrode conditioning (cleaning from the previous measurement) applying $+0.5 \mathrm{~V}$ for $30 \mathrm{~s}$ under stirring at $1750 \mathrm{rpm}$; (ii) electrodeposition of metals applying $-1.0 \mathrm{~V}$ for $180 \mathrm{~s}$ under stirring at $1750 \mathrm{rpm}$; and (iii) electrochemical stripping away of metals by scanning the electrode from -0.7 to $+0.2 \mathrm{~V}$ (frequency: $30 \mathrm{~Hz}$, amplitude: $90 \mathrm{mV}$; step potential: $9 \mathrm{mV}$ ) using unstirred solution. The concentration of both metals was varied from 200 to $1200 \mu \mathrm{g} \mathrm{L}^{-1}$ for both metals. Figure 2 
shows the current increase as function of concentration of both metals.

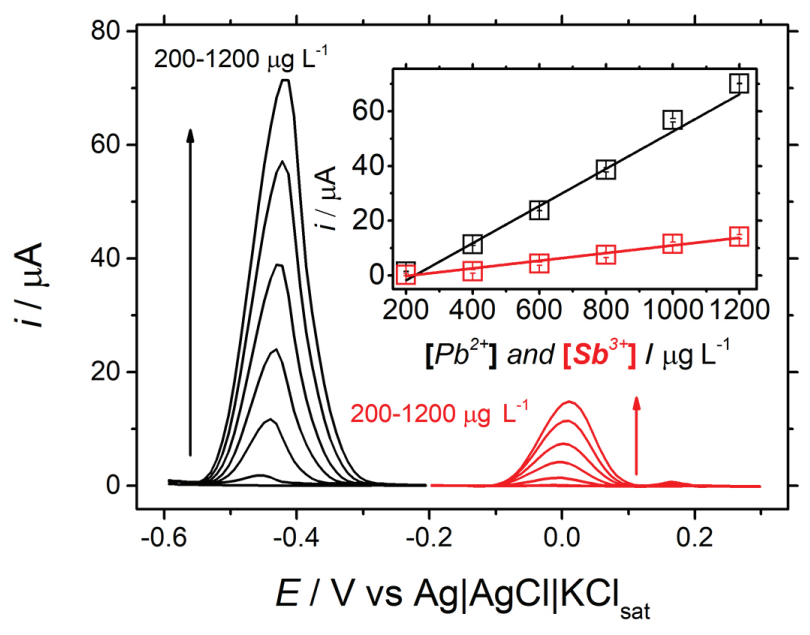

Figure 2. SWASV responses for the study of linear range (from 200 to $1200 \mu \mathrm{g} \mathrm{L}^{-1}$ for $\mathrm{Pb}^{2+}$ in black and $\mathrm{Sb}^{3+}$ in red) and (inset) respective calibrations curves $\left(\mathrm{Pb}^{2+}\right.$ : black squares; $\mathrm{Sb}^{3+}$ : red squares). Experimental conditions: $0.01 \mathrm{~mol} \mathrm{~L}^{-1} \mathrm{HCl}$ solution (supporting electrolyte); $90 \mathrm{mV}$ (amplitude); $30 \mathrm{~Hz}$ (frequency); $9 \mathrm{mV}$ (step potential); -1.0 V (deposition potential); $180 \mathrm{~s}$ (deposition time) and 1750 (stirring rate). Electrode design shown in Figure 1Ba filled with extruded graphene/PLA filament; reference electrode: $\mathrm{Ag}|\mathrm{AgCl}| \mathrm{KCl}_{\text {sat }}$.

This result is a promising result that illustrate one potential application of such sensors to the voltammetric stripping determination of metals. One great advantage of this sensor is the absence of chemical modifiers (e.g., metallic films of $\mathrm{Hg}$ or $\mathrm{Bi}$ ) using a carbon-based electrode. Glassy-carbon as well as other carbon based electrodes are typically modified with $\mathrm{Hg}^{38}$ or $\mathrm{Bi}^{39}$ films to overcome the hydrogen evolution, enabling the application of $-1.0 \mathrm{~V}$ ( vs. $\left.\mathrm{Ag}|\mathrm{AgCl}| \mathrm{KCl}_{\text {sat }}\right)$ in acidic media for the successful determination of metals by stripping analysis. Previous works ${ }^{9,40}$ using 3D-printed conductive PLA electrodes suggested that the carboxyl functional groups of the PLA matrix may work as chelating agents of metallic ions, improving metal detection after preconcentration without the need for surface modification.

Another interesting feature of this graphene/PLA electrode to be explored is its application to generate chemically-modified electrodes, for instance to obtain Prussian Blue (iron hexacyanoferrate) modified electrodes. ${ }^{41}$ Commercial graphene/PLA filaments may contain iron impurities as recently reported ${ }^{42}$ and this iron source can be used to electrochemically synthesize iron complexes at the graphene/PLA substrate after continuous cyclic voltammetric scans as demonstrated in the literature, ${ }^{43}$ inspired in the work by Nossol and Zarbin ${ }^{44}$ that synthesized Prussian Blue from iron impurities present in carbon nanotubes. Figure S1a (SI section) shows the cyclic voltammograms $\left(1^{\text {st }}, 50^{\text {th }}, 100^{\text {th }}, 150^{\text {th }}\right.$ and $200^{\text {th }}$ cycles $)$ in the presence of ferricyanide. This first cycle shows a typical voltammetric profile for the redox ferricyanide/ ferrocyanide pair, except for the high value of peak-to-peak separation $(\Delta \mathrm{E}$, difference between the oxidation and reduction peak potentials) of around $200 \mathrm{mV}$. This value is far from the ideal $59 \mathrm{mV}$ for the mono-electron transfer of this electrochemically reversible system; however, it has to be considered that the working electrode is made of a thermoplastic material that hinders the electron transfer in comparison to a glassy-carbon electrode. ${ }^{34}$ It is noteworthy to mention that after 200 cycles it is possible to see the electrochemical growth of a new redox pair close to $+0.85 \mathrm{~V}$ corresponding to one of the iron hexacyanoferrate (Prussian Blue, PB) processes. To confirm the formation of the PB films, the same electrode was rinsed with deionized water and immersed in the supporting electrolyte solution $\left(0.1 \mathrm{~mol} \mathrm{~L}^{-1} \mathrm{KCl}+0.01 \mathrm{~mol} \mathrm{~L}^{-1} \mathrm{HCl}\right)$ in absence of ferricyanide. Figure S1b (SI section) shows this cyclic voltammogram which show the corresponding two redox pair processes typically found for PB films at around +0.2 and $+0.9 \mathrm{~V}$. The dashed line shows the cyclic voltammogram in absence of the Prussian Blue film (bare graphene/PLA electrode) to highlight the formation of PB films by performing cyclic voltammograms in the presence of ferricyanide. Hence, this simple protocol can be performed to generate a highly selective sensor for strong oxidants, ${ }^{45}$ such as hydrogen peroxide and free chlorine, with potential applications in a wide range of samples. Figure S1c (SI section) shows the amperometric recording of a stirred solution after successive additions of $100 \mu \mathrm{mol} \mathrm{L} \mathrm{L}^{-1} \mathrm{H}_{2} \mathrm{O}_{2}$ and Figure S1d (SI section) shows the respective calibration curve. The current in Figure $\mathrm{S} 1 \mathrm{c}$ decreased after each addition of $\mathrm{H}_{2} \mathrm{O}_{2}$ due to the electrocatalytic action of the PB-modified surface towards the electrochemical reduction of $\mathrm{H}_{2} \mathrm{O}_{2}$. The low applied potential $\left(0.0 \mathrm{~V} v s . \mathrm{Ag}|\mathrm{AgCl}| \mathrm{KCl}_{\mathrm{sat}}\right)$ is a characteristic of $\mathrm{PB}$ modified electrodes that consequently provides great selectivity for $\mathrm{H}_{2} \mathrm{O}_{2}$ detection, as other molecules do not present an electrochemical response in this condition. Moreover, the sensor provided a wide linear range.

Figure 3 shows cyclic voltammograms in the presence of ferricyanide using the device shown in Figure $1 \mathrm{Bb}$ that contains the three electrodes in a tube. In this case, a carbon black/PLA filament was extruded by the 3D pen on the three pockets corresponding to the working, counter and reference electrodes. The voltammogram in red was recorded using a carbon black/PLA as pseudoreference electrode while in the voltammogram in blue the reference was replaced by an external $\mathrm{Ag}|\mathrm{AgCl}| \mathrm{KCl}_{\text {sat }}$ reference electrode. 


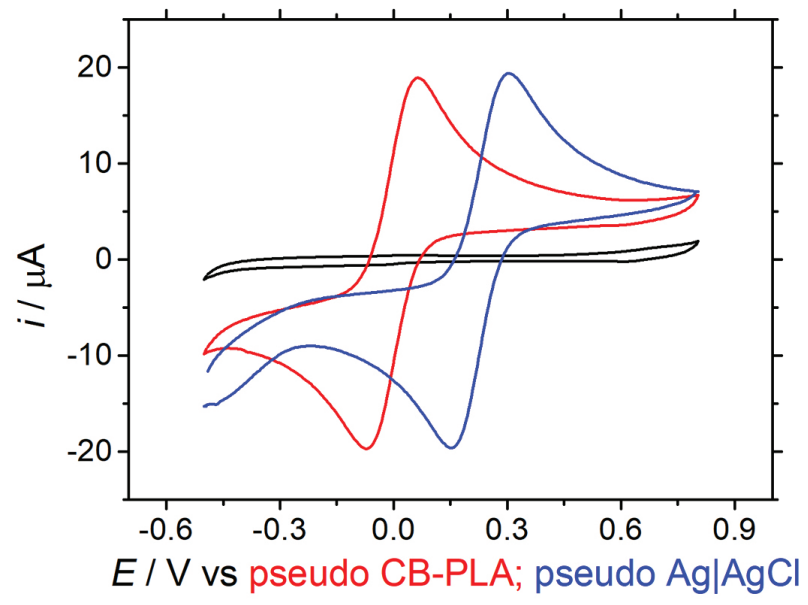

Figure 3. Cyclic voltammograms at $0.1 \mathrm{~mol} \mathrm{~L}^{-1}$ of $\mathrm{KCl}$ as electrolyte of blank solution (-) and after the addition of $1 \mathrm{mmol} \mathrm{L}^{-1}\left[\mathrm{Fe}(\mathrm{CN})_{6}\right]^{3-/ 4-}(1: 1)$ using an external $\mathrm{Ag}|\mathrm{AgCl}| \mathrm{KCl}_{\text {sat }}(-)$ and a pseudo carbon black/PLA $(-)$ reference electrode. Electrode design shown in Figure $1 \mathrm{Bb}$ filled with extruded carbon black/PLA filament.

Both voltammograms show the typical profile for the redox probe $\left[\mathrm{Fe}(\mathrm{CN})_{6}\right]^{3-14-}$ with a $\Delta \mathrm{E}$ value of $150 \mathrm{mV}$, which is slightly lower than that obtained on the graphene/ PLA electrode. The shift of potential observed of both redox processes is due to the potential of the reference electrode. This simple experiment shows that it is possible to use a carbon/black pseudo-reference in the devices containing the three electrodes fabricated using the same thermoplastic material using the 3D pen. Nevertheless, some authors ${ }^{29}$ reported the lack of stability of the potential of carbonbased pseudo-reference electrodes under continuous electrochemical measurements which can generate the shift of oxidation/reduction peaks in the voltammograms. This potential shift is more severe considering the amperometric detection technique in which the precise control of the applied potential is required for accurate amperometric recordings. ${ }^{46,47}$ For this reason, it was proposed the modification of a carbon black pseudo-reference electrode into an $\mathrm{Ag} / \mathrm{AgCl}$ pseudo-reference electrode to increase the stability of the measurements.

Next experiments, presented in Figure 4, were obtained using the device shown in Figure $1 \mathrm{Bc}$ in which a carbon black/PLA filament was extruded by the 3D pen on the three pockets corresponding to the working, counter and reference electrodes. The reference electrode was modified as described in the Experimental section to generate the $\mathrm{Ag} / \mathrm{AgCl}$ pseudo-reference electrode. Figure 4 shows square-wave voltammetric (SWV) recordings for increasing concentrations of dopamine and the inset shows the corresponding calibration curve, in which it is possible to see two different linear concentration ranges (from 1 to 70 and from 95 to $250 \mu \mathrm{mol} \mathrm{L}-1$ ). Dopamine is a relevant catecholamine neurotransmitter with important functions in the brain and due to its electroactivity it has been selected as a model analyte. The 3D pen fabricated sensor is capable to detect submicromolar concentrations of dopamine under the selected conditions, which shows great promises for applications in biological fluids. Dopamine sensing on 3D printed graphene/PLA electrodes using desktop 3D printers was reported in the literature, ${ }^{12}$ and the authors also found two linear ranges, from 5 to 100 and from 100 to $1000 \mu \mathrm{mol} \mathrm{L}^{-1}$. The treatment of the electrode surface was slightly different, consisting of immersion in DMF and electrochemical treatment in $\mathrm{NaOH}$. Even though, the same behavior for dopamine detection using SWV was verified with two linear curves which is an indicative of high competition of the electroactive species for the electrode surface.

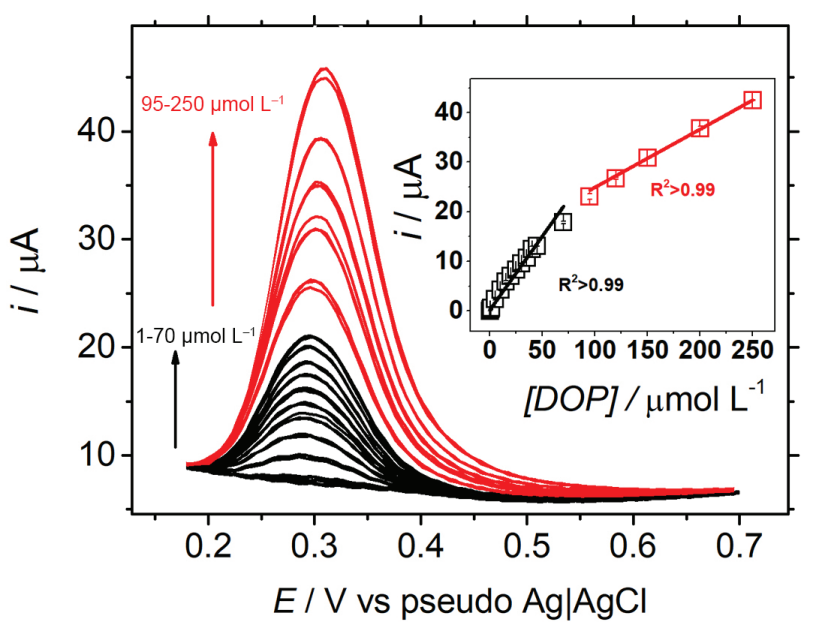

Figure 4. SWV recordings of triplicate scans of crescent concentrations of dopamine. Two linear concentration ranges are highlighted: 1 to 70 (in black) and 95 to $250 \mu \mathrm{mol} \mathrm{L}-1$ (in red). The inset corresponds to the respective analytical curves $\left(\mathrm{R}^{2}>0.99\right)$. SWV parameters: step potential of $5 \mathrm{mV}$, amplitude of $50 \mathrm{mV}$, frequency of $50 \mathrm{~Hz}$; supporting electrolyte: $0.1 \mathrm{~mol} \mathrm{~L}^{-1} \mathrm{HClO}_{4}$; electrode design shown in Figure $1 \mathrm{Bc}$ filled with extruded carbon black/PLA filament; reference electrode: pseudo $\mathrm{Ag} \mid \mathrm{AgCl}$.

Figure S2a (SI section) shows SWV recordings obtained in the devices shown in Figures $1 \mathrm{Bb}$ and $1 \mathrm{Bc}$ using different reference electrodes (3D-printed carbon black/PLA and $\mathrm{Ag} / \mathrm{AgCl}$ pseudo-reference, and external $\mathrm{Ag}|\mathrm{AgCl}| \mathrm{KCl}_{\text {sat }}$ reference electrodes). It is possible to verify the potential shift according to the reference electrode used in each case; however, this effect was not a drawback in the obtaining of an analytical curve for dopamine using standard solutions in the same concentration range (from 1 to $100 \mu \mathrm{mol} \mathrm{L}^{-1}$ ). Figure S2b (SI section) shows the corresponding calibration curves which present similar slope values $(0.228,0.286$ and $0.260 \mu \mathrm{A} \mu \mathrm{mol}^{-1} \mathrm{~L}$ using pseudo carbon black/PLA, pseudo $\mathrm{Ag} / \mathrm{AgCl}$ and $\mathrm{Ag}|\mathrm{AgCl}| \mathrm{KCl}_{\text {sat }}$ reference electrodes). Hence, the proposed electrochemical devices using thermoplastic 
conductive electrodes fabricated using a 3D pen and prototyped in different designs offer great promises for electrochemical sensing of several analytes in a wide range of applications, including the analysis of low volumes (e.g., single-drop analysis) using the planar device with threeelectrodes (Figure 1Bc).

\section{Conclusions}

We have demonstrated how a 3D pen can be used to draw thermoplastic electrodes using commercial conductive PLA filaments. Commercial PLA filaments containing carbon black or graphene can be used in the 3D pen to extrude the electrodes. Lab-made conductive filaments using recycled polymers can decrease even more the cost for obtaining the electrodes using the 3D pen. We presented three different designs in which a low-cost desktop FDM 3D-printer was used to fabricate the customized electrode susbtrates; however, many other materials can be applied as a template to fabricate the thermoplastic electrodes. Applications of such electrodes can go beyond electrochemical sensors, extending to batteries or supercapacitors if filaments with superior conductivity are employed. Additionally, the affordability and safe use of a 3D pen enables the fabrication of electrodes for teaching purposes using low consumables and materials.

\section{Supplementary Information}

Supplementary information (Figures S1 and S2) is available free of charge at http://jbcs.sbq.org.br as PDF file. A video showing the step-by-step fabrication of the device shown in Figure 1Bc is also available.

\section{Acknowledgments}

The authors are grateful to CNPq (427731/2018-6 and 307271/2017-0), FAPEMIG (RED-00042-16 and APQ03141-18), CAPES (001), and INCTBio (CNPq grant No. 465389/2014-7) for financial support.

\section{References}

1. Ambrosi, A.; Pumera, M.; Chem. Soc. Rev. 2016, 45, 2740.

2. Symes, M. D.; Kitson, P. J.; Yan, J.; Richmond, C. J.; Cooper, G. J. T.; Bowman, R. W.; Vilbrandt, T.; Cronin, L.; Nat. Chem. 2012, 4, 349.

3. Rogers, C. I.; Qaderi, K.; Woolley, A. T.; Nordin, G. P.; Biomicrofluidics 2015, 9, 016501.

4. Foo, C. Y.; Lim, H. N.; Mahdi, M. A.; Wahid, M. H.; Huang, N. M.; Sci. Rep. 2018, 8, 7399.
5. Zhu, C.; Han, T. Y. J.; Duoss, E. B.; Golobic, A. M.; Kuntz, J. D.; Spadaccini, C. M.; Worsley, M. A.; Nat. Commun. 2015, 6, 6962 .

6. Pang, Y.; Cao, Y.; Chu, Y.; Liu, M.; Snyder, K.; MacKenzie, D.; Cao, C.; Adv. Funct. Mater. 2020, 30, 1906244.

7. Hughes, J. P.; Santos, L.; Down, M. P.; Foster, C. W.; Bonacin, J. A.; Keefe, E. M.; Rowley-Neale, S. J.; Banks, C. E.; Sustainable Energy Fuels 2020, 4, 302.

8. Manzanares Palenzuela, C. L.; Novotný, F.; Krupička, P.; Sofer, Z.; Pumera, M.; Anal. Chem. 2018, 90, 5753.

9. Rocha, D. P.; Squissato, A. L.; da Silva, S. M.; Richter, E. M.; Munoz, R. A. A.; Electrochim. Acta 2020, 335, 135688.

10. O’Neil, G. D.; Ahmed, S.; Halloran, K.; Janusz, J. N.; Rodríguez, A.; Terrero Rodríguez, I. M.; Electrochem. Commun. 2019, 99, 56.

11. Katseli, V.; Economou, A.; Kokkinos, C.; Electrochem. Commun. 2019, 103, 100.

12. Kalinke, C.; Neumsteir, N. V.; Aparecido, G. O.; Ferraz, T. V. B.; dos Santos, P. L.; Janegitz, B. C.; Bonacin, J. A.; Analyst 2020, 145, 1207.

13. Honeychurch, K. C.; Rymansaib, Z.; Iravani, P.; Sens. Actuators, B 2018, 267, 476.

14. Vaněčková, E.; Bouša, M.; Lachmanová, Š. N.; Rathouský, J.; Gál, M.; Sebechlebská, T.; Kolivoška, V.; J. Electroanal. Chem. 2020, 857, 113745.

15. João, A. F.; Squissato, A. L.; Fernandes, G. M.; Cardoso, R. M.; Batista, A. D.; Muñoz, R. A. A.; Microchem. J. 2019, 146, 1134.

16. Escobedo, P.; Palma, A. J.; Erenas, M. M.; Martinez Olmos, A.; Carvajal, M. A.; Tabraue Chavez, M.; Luque Gonzalez, M. A.; Diaz-Mochon, J. J.; Pernagallo, S.; Capitan-Vallvey, L. F.; IEEE Access 2019, 7, 185677.

17. Ambrosi, A.; Pumera, M.; Adv. Funct. Mater. 2018, 28, 1700655 .

18. Zambiazi, P. J.; de Moraes, A. T. N.; Kogachi, R. R.; Aparecido, G. O.; Formiga, A. L. B.; Bonacin, J. A.; J. Braz. Chem. Soc. in press, DOI 10.21577/0103-5053.20200088.

19. Swennen, G. R. J.; Pottel, L.; Haers, P. E.; Int. J. Oral Maxillofac. Surg. 2020, 49, 673.

20. Adams, A.; Malkoc, A.; La Belle, J. T.; J. Diabetes Sci. Technol. 2018, 12, 176.

21. dos Santos, P. L.; Katic, V.; Loureiro, H. C.; dos Santos, M. F.; dos Santos, D. P.; Formiga, A. L. B.; Bonacin, J. A.; Sens. Actuators, B 2019, 281, 837.

22. Rocha, D. P.; Foster, C. W.; Munoz, R. A. A.; Buller, G. A.; Keefe, E. M.; Banks, C. E.; Analyst 2020, 145, 3424.

23. Foster, C. W.; Elbardisy, H. M.; Down, M. P.; Keefe, E. M.; Smith, G. C.; Banks, C. E.; Chem. Eng. J. 2020, 381, 122343.

24. Maurel, A.; Courty, M.; Fleutot, B.; Tortajada, H.; Prashantha, K.; Armand, M.; Grugeon, S.; Panier, S.; Dupont, L.; Chem. Mater. 2018, 30, 7484. 
25. Maurel, A.; Grugeon, S.; Fleutot, B.; Courty, M.; Prashantha, K.; Tortajada, H.; Armand, M.; Panier, S.; Dupont, L.; Sci. Rep. 2019, 9, 18031.

26. Sanatgar, R. H.; Cayla, A.; Campagne, C.; Nierstrasz, V.; J. Appl. Polym. Sci. 2019, 136, 47040.

27. de Araujo, F. P.; Batista, I. T. S.; de Oliveira, F. C.; de Almeida, L. R.; Brito, G. C.; Barud, H. S.; Dittz, D.; Silva-Filho, E. C.; Osajima, J. A.; Lobo, A. O.; J. Mater. Res. 2020, 35, 1154.

28. Sousa, L. R.; Duarte, L. C.; Coltro, W. K. T.; Sens. Actuators, B 2020, 312, 128018.

29. Rohaizad, N.; Mayorga-Martinez, C. C.; Novotný, F.; Webster, R. D.; Pumera, M.; Electrochem. Commun. 2019, 103, 104.

30. Cardoso, R. M.; Mendonça, D. M. H.; Silva, W. P.; Silva, M. N. T.; Nossol, E.; da Silva, R. A. B.; Richter, E. M.; Muñoz, R. A. A.; Anal. Chim. Acta 2018, 1033, 49.

31. Pedrotti, J. J.; Angnes, L.; Gutz, I. G. R.; Electroanalysis 1996, $8,673$.

32. Cardoso, R. M.; Silva, P. R. L.; Lima, A. P.; Rocha, D. P.; Oliveira, T. C.; do Prado, T. M.; Fava, E. L.; Fatibello-Filho, O.; Richter, E. M.; Muñoz, R. A. A.; Sens. Actuators, B 2020, 307, 127621.

33. Richter, E. M.; Rocha, D. P.; Cardoso, R. M.; Keefe, E. M.; Foster, C. W.; Munoz, R. A. A.; Banks, C. E.; Anal. Chem. 2019, 91, 12844.

34. Cardoso, R. M.; Kalinke, C.; Rocha, R. G.; dos Santos, P. L.; Rocha, D. P.; Oliveira, P. R.; Janegitz, B. C.; Bonacin, J. A.; Richter, E. M.; Munoz, R. A. A.; Anal. Chim. Acta 2020, 1118 , 73.

35. Browne, M. P.; Novotný, F.; Sofer, Z.; Pumera, M.; ACS Appl. Mater. Interfaces 2018, 10, 40294.
36. Cardoso, R. M.; Castro, S. V. F.; Silva, M. N. T.; Lima, A. P.; Santana, M. H. P.; Nossol, E.; Silva, R. A. B.; Richter, E. M.; Paixão, T. R. L. C.; Muñoz, R. A. A.; Sens. Actuators, B 2019, 292, 308 .

37. Novotný, F.; Urbanová, V.; Plutnar, J.; Pumera, M.; ACS Appl. Mater. Interfaces 2019, 11, 35371.

38. Munoz, R. A. A.; Silva, C. S.; Correia, P. R. M.; Oliveira, P. V.; Angnes, L.; Microchim. Acta 2005, 149, 199.

39. Švancara, I.; Prior, C.; Hočevar, S. B.; Wang, J.; Electroanalysis 2010, 22, 1405.

40. João, A. F.; Squissato, A. L.; Richter, E. M.; Muñoz, R. A. A.; Anal. Bioanal. Chem. 2020, 412, 2755.

41. Katic, V.; dos Santos, P. L.; dos Santos, M. F.; Pires, B. M.; Loureiro, H. C.; Lima, A. P.; Queiroz, J. C. M.; Landers, R.; Muñoz, R. A. A.; Bonacin, J. A.; ACS Appl. Mater. Interfaces 2019, 11, 35068.

42. Browne, M. P.; Urbanova, V.; Plutnar, J.; Novotný, F.; Pumera, M.; J. Mater. Chem. A 2020, 8, 1120.

43. Rocha, R. G.; Santos, J. S.; Cardoso, R. C.; Zambiazi, P. J.; Bonacin, J. A.; Richter, E. M.; Munoz, R. A. A.; Talanta, in press, DOI 10.1016/j.talanta.2020.121289.

44. Nossol, E.; Zarbin, A. J. G.; Adv. Funct. Mater. 2009, 19, 3980.

45. Silva, S. C.; Cardoso, R. M.; Richter, E. M.; Munoz, R. A. A.; Nossol, E.; Mater. Chem. Phys. 2020, 250, 123011.

46. dos Santos, W. T. P.; Gimenes, D. T.; Richter, E. M.; Angnes, L.; Quim. Nova 2011, 34, 1753.

47. Rocha, D. P.; Cardoso, R. M.; Tormin, T. F.; de Araujo, W. R.; Munoz, R. A. A.; Richter, E. M.; Angnes, L.; Electroanalysis 2018, 30, 1386. 